\title{
Hypolipidemic effects of HVC1 in a high cholesterol diet-induced rat model of hyperlipidemia
}

\author{
CHAE-YUN KIM ${ }^{1 *}$, KYUNG-SOOK CHUNG ${ }^{1 *}$, SE-YUN CHEON ${ }^{1}$, KYUNGJIN LEE $^{2}$, INHYE HAM ${ }^{2}$, \\ HO-YOUNG CHOI ${ }^{2}$, YONG BAIK $\mathrm{CHO}^{3}$, BYOUNG-HEON CHO ${ }^{3}$, SO YOUN MOK ${ }^{3}$ and HYO-JIN AN ${ }^{1}$ \\ ${ }^{1}$ Department of Pharmacology, College of Oriental Medicine, Sangji University, Wonju, Gangwon-do 220702; \\ ${ }^{2}$ Department of Herbology, College of Korean Medicine, Kyung Hee University, Seoul 130701; \\ ${ }^{3}$ Pharmaceutical R\&D Center, Kolmar Korea Co. Ltd, Sejong 339853, Republic of Korea
}

Received August 10, 2015; Accepted July 7, 2016

DOI: $10.3892 / \mathrm{mmr} .2016 .5615$

\begin{abstract}
HVC1, a novel formation containing four herbs, was developed and its hypolipidemic effects in rats with high cholesterol diet (HCD)-induced hyperlipidemia were investigated. The rats were given a HCD for 8 weeks. The HVC1-treated groups were orally administered $\mathrm{HVCl}$ at doses of 10,50 or $250 \mathrm{mg} / \mathrm{kg}$, respectively, and the simvastatin group was treated at a dose of $10 \mathrm{mg} / \mathrm{kg}$. The normal diet and HCD control groups were administered with physiological saline. Oral administration of HVC1 $(10,50$ or $250 \mathrm{mg} / \mathrm{kg})$ significantly reduced the body weight of rats with hyperlipidemia and regulated the total cholesterol, low-density lipoprotein cholesterol and high-density lipoprotein cholesterol levels in the serum. In addition, tissue analysis revealed that lipid accumulation in the liver and aorta was reduced by $\mathrm{HVC1}$ administration. Furthermore, HVC1 significantly reduced the mRNA expression of peroxisome proliferator-activated receptor- $\gamma, 3$-hydroxy-3-methylglutaryl-CoA reductase and low-density lipoprotein receptor, as well as the protein level of $5^{\prime}$ adenosine monophosphate-activated protein kinase in the liver. The results clearly demonstrate that $\mathrm{HVC1}$ has a potent hypolipidemic effect, and suggest that $\mathrm{HVC1}$ should be evaluated as a potential treatment for hyperlipidemia.
\end{abstract}

\section{Introduction}

Hyperlipidemia is a group of disorders characterized by elevated concentrations of circulating lipids, including cholesterol, cholesterol esters, phospholipids and triglycerides.

Correspondence to: Professor Hyo-Jin An, Department of Pharmacology, College of Oriental Medicine, Sangji University, 83 Sangjidae-gil, Wonju, Gangwon-do 220702, Republic of Korea E-mail: hjan@sj.ac.kr

*Contributed equally

Key words: hyperlipidemia, high cholesterol diet, cholesterol, lipid metabolism, $\mathrm{HVCl}$
It often results from delayed or defective clearance, or overproduction of very low-density lipoprotein (VLDL) by the liver, which is subsequently transformed into low-density lipoprotein (LDL). Excessive intake of saturated fats increases lipid production in the liver via a molecular mechanism involving protein activators (1). Notably, hyperlipidemia is a major modifiable risk factor for atherosclerosis and cardiovascular disease, including coronary heart disease.

In the present study, 4 herbs were combined to form $\mathrm{HVC1}$, based on the theory of Korean medicine. HVC1 is composed of Prunus yedoensis bark (Rosaceae), Rheum palmatum rhizome (Polygonaceae), Coptis chinensis rhizome (Ranunculaceae) and Scutellaria baicalensis radix (Lamiaceae). $P$. yedoensis bark (Rosaceae) is a traditional medicine used to treat cough, urticaria, pruritus, dermatitis, asthma and measles $(2,3)$. It has been reported that $P$. yedoensis bark induced vasorelaxation in isolated rat thoracic aorta rings through increased nitric oxide (NO) formation (4). Additionally, it was demonstrated that prunetin, a component of $P$. yedoensis bark, efficiently suppressed adipogenesis and obesity, as well as serum lipid levels (5). R. palmatum is used most frequently in the weight-reducing formulae of traditional Chinese medicine. Recently, rhein, one of the major components of Rheum palmatum, has been shown to be an inhibitor of 3T3-L1 adipocyte differentiation (6). Moreover, rhein has been reported to have pharmacological and biochemical effects on the inhibition of liver fibrosis and insulin sensitization (7-9), and to prevent hepatic steatosis through LXR inhibition in a high-fat diet-induced obese mouse model (10). It has been reported that $C$. chinensis rhizome and its primary active component, berberine, have anti-obesity activity. C. chinensis rhizome and berberine significantly reduce blood glucose and lipid levels in high-fat diet-fed mice and increase mRNA expression of AMP-activated protein kinase (AMPK) to promote mitochondrial energy metabolism (11). $R$. palmatum rhizome and anthraquinones significantly reduce blood levels of total cholesterol (TC), triglycerides, high-density lipoprotein (HDL) and LDL (12). S. baicalensis radix protects against high fat and alcoholic liver damage through the inhibition of 3-hydroxy-3-methylglutaryl-CoA reductase (HMG-CoA R) expression in the liver (13). In addition, the primary active compounds of $S$. baicalensis 
radix, baicalein and wogonin have been reported to attenuate obesity, dyslipidemia and diabetes (14).

Based on previous data, HVC1 was developed to treat or prevent hyperlipidemic diseases (15) and the present study was designed to examine the hypolipidemic effects of $\mathrm{HVC} 1$ in a high cholesterol diet (HCD)-induced hyperlipidemia rat model.

\section{Materials and methods}

Reagents. The normal diet (ND) and HCD were obtained from Research Diets (New Brunswick, NJ, USA). The P-5' adenosine monophosphate-activated protein kinase (P-5' AMPK; Thr 172; cat. no. sc-33524), AMPK (cat. no. sc-398861) and $\beta$-actin (cat. no. sc-81178) monoclonal antibodies were purchased from Santa Cruz Biotechnology Inc. (Santa Cruz, CA, USA). Horseradish peroxidase-conjugated anti-mouse (cat. no. 315-035-003) and anti-rabbit (cat. no. 111-035-003) were purchased from Jackson ImmunoResearch Laboratories, Inc. (West Grove, PA, USA). All other reagents were purchased from Sigma-Aldrich (St. Louis, MO, USA).

Preparation of HVCl. HVC1 consists of $P$. yedoensis Matsum bark, $R$. palmatum L. rhizome, $C$. chinensis Franch. rhizome and $S$. baicalensis Georgi radix. The herbs were purchased from Dongwoodang Co., Ltd. (Yeongcheon, Korea). Professor Ho-Young Choi of Kyung Hee University identified the plants. Voucher specimens of $C$. chinensis rhizome (CC001), $S$. baicalensis radix (SB001), R. palmatum rhizome (RP001), and $P$. yedoensis bark (PY001) were deposited at the College of Korean Medicine, Kyung Hee University (Seoul, Korea). Each herb was used at a ratio of 2:2:1:1 (600:600:300:300 g). The herbs were extracted using $30 \%(\mathrm{v} / \mathrm{v})$ ethanol in water at $60^{\circ} \mathrm{C}$ for $8 \mathrm{~h}$. The extracts were filtered through $10-\mu \mathrm{m}$ cartridge paper, and the ethanol was removed by vacuum rotary evaporation (EYELA; Tokyo, Japan). The concentrates were freeze-dried, and the yield was calculated to be $13 \%$. The powders were dissolved in distilled water for the experiments, and the residual powder was stored at $-20^{\circ} \mathrm{C}$.

Animal experiments. Sprague-Dawley (SD) rats $(n=36$; 4 weeks, male, 200-230 g) were purchased from Daehan Biolink (Daejeon, Korea) and maintained under constant conditions (temperature, $20-25^{\circ} \mathrm{C}$; humidity, $40-60 \% ; 12 \mathrm{~h}$ light/dark cycle). The rats were adapted to the feeding conditions for 1 week and then given free access to food and tap water for 8 weeks. The rats were randomly assigned to 6 groups (n=6): ND group, HCD group, and treatment groups fed HCD with simvastatin $(10 \mathrm{mg} / \mathrm{kg})$ or HVC1 $(10,50$ or $250 \mathrm{mg} / \mathrm{kg}$ ). With the exception of the ND group, all of the rats consumed a HCD (D12108) containing 20.1\% saturated fat, $1.37 \%$ cholesterol and $0 \%$ sodium cholate. HVC1 was orally administered to the treated groups, and the untreated groups received the same volume $(500 \mu \mathrm{l})$ of physiological saline for 8 weeks. Body weight and dietary intake were recorded every week. After 8 weeks, the animals were fasted overnight. The following day, they were anesthetized with Zoletil (50 mg/kg; Virbac; Carros Cedex, France), and blood samples were collected by cardiac puncture using a $3 \mathrm{ml}$ syringe with $23 \mathrm{G}$ needle. Immediately following collection of blood sample, the rats were sacrificed by exsanguination under anesthesia with Zoletil $(60 \mathrm{mg} / \mathrm{kg})$. To remove clots, whole blood were separated by centrifugation at $1,200 \mathrm{x} \mathrm{g}$, for $10 \mathrm{~min}\left(4^{\circ} \mathrm{C}\right)$ and supernatants were maintained at $2-8^{\circ} \mathrm{C}$. The liver and aorta were excised, rinsed, weighed and directly stored at $-80^{\circ} \mathrm{C}$ until analyzed. All procedures were conducted in accordance with the National Institutes of Health guidelines and approved by the Ethical Committee for Animal Care and the Use of Laboratory Animals, Sangji University (Wonju, Korea; reg. no. 2014-05).

Serum analysis. Serum concentrations of TC, LDL cholesterol, and HDL cholesterol were determined by enzymatic methods with HDL and LDL/VLDL Quantification Colorimetric/Fluorometric kit (cat. no. K613-100; BioVision, Milpitas, CA, USA).

Histological analysis. The liver and aorta from a representative rat in each group were fixed in $10 \%$ buffered formalin, embedded in paraffin, and cut into $5-\mu \mathrm{m}$ sections. Certain sections were stained with hematoxylin and eosin (H\&E) for histological examination of fat droplets. Samples of aorta were stained with Oil red O, as previously described (16). Images were acquired using an SZX10 microscope (Olympus; Tokyo, Japan).

Western blot analysis. Liver tissues were homogenized in the protein extraction solution, PRO-PREP (Intron Biotechnology, Gyeongi-do, Korea), and then incubated for $25 \mathrm{~min}$ on ice. The samples were centrifuged at $16,000 \mathrm{x} \mathrm{g}\left(4^{\circ} \mathrm{C}\right)$ for $5 \mathrm{~min}$, and the supernatant was transferred to a new $1.5-\mathrm{ml}$ tube. The protein concentration was determined using the Bio-Rad protein assay reagent, according to the manufacturer's instructions (Bio-Rad Laboratories, Hercules, CA, USA). Protein samples were immunoblotted onto a polyvinylidene difluoride (PVDF) membrane following separation on a $10 \%$ sodium dodecyl sulfate (SDS)-polyacrylamide gel. The membranes were blocked with a 5\% skimmed milk solution for $1 \mathrm{~h}$ and incubated overnight with a primary antibody (1:1,000 dilution). The immunoblots were washed 3 times with Tween 20/Tris-buffered saline (TTBS) and incubated with the corresponding secondary antibody (1:2,000 dilution) for $1 \mathrm{~h}$ at room temperature. After washing 3 times with TTBS, the immunoblots were developed using enhanced chemiluminescence and X-ray film (Amersham Life Science, Buckinghamshire, UK).

Reverse transcription-quantitative polymerase chain reaction $(R T-q P C R)$. The liver tissues were homogenized, and total RNA was isolated using the Easy-Blue Reagent according to the manufacturer's instructions (Intron Biotechnology). Total RNA was quantified using an Epoch micro-volume spectrophotometer system (BioTek Instruments, Winooski, VT, USA). In brief, total RNA was converted to cDNA using a high-capacity cDNA reverse transcription kit (Applied Biosystems, Foster City, CA, USA) and thermocycler (Gene Amp PCR system 9700; Applied Biosystems) with the following program: Initiation for $10 \mathrm{~min}$ at $25^{\circ} \mathrm{C}$, followed by incubation at $50^{\circ} \mathrm{C}$ for $90 \mathrm{~min}$ and at $85^{\circ} \mathrm{C}$ for 5 min. qPCR analysis was conducted using a Step One Plus Real-time PCR system (StepOne software version 2.3; 
Table I. Primer sequences.

\begin{tabular}{lcc}
\hline Gene name & Forward primer & Reverse primer \\
\hline PPAR- $\gamma$ & 5'-ATCGAGTGCCGAGTCTGTGG-3' & 5'-GCAAGGCACTTCTGAAACCG-3' \\
HMG-CoA R & 5'-TGTTGGAGTGGCAGGACCTC-3' & 5'-GGCACCTCCACCAAGACTGA-3' \\
LDL receptor & 5'-CTCACTTCCGCTGCAACTCC-3' & 5'-CCACAGTGGAACTCGAGGGA-3' \\
GAPDH & 5'-TGATTCTACCCACGGCAAGT-3' & 5'-AGCATCACCCCATTTGATGT-3'
\end{tabular}

PPAR- $\gamma$ HMG-CoA R LDL receptor PPAR- $\gamma$, peroxisome proliferator-activated receptor- $\gamma$; HMG-CoA R, 3-hydroxy-3-methylglutaryl-CoA reductase; LDLR, low-density lipoprotein receptor; AMPK, AMP-activated protein kinase; p-, phosphorylated.

Applied Biosystems). SYBR Green master mix and primers were used for PCR analysis of glyceraldehyde-3-phosphate dehydrogenase (GAPDH), peroxisome proliferator-activated receptor- $\gamma$ (PPAR- $\gamma)$, HMG-CoA R and low-density lipoprotein receptor (LDLR). The PCR cycling parameters were as follows: $10 \mathrm{~min}$ at $95^{\circ} \mathrm{C} ; 40$ cycles of $5 \mathrm{sec}$ at $95^{\circ} \mathrm{C}$ and $45 \mathrm{sec}$ at $60^{\circ} \mathrm{C}$; and a final melting curve of $15 \mathrm{sec}$ at $95^{\circ} \mathrm{C}, 1 \mathrm{~min}$ at $60^{\circ} \mathrm{C}$, and $15 \mathrm{sec}$ at $95^{\circ} \mathrm{C}$. All primer sequences are shown in Table I. Gene expression was calculated according to the Cq method.

High Performance Liquid Chromatography (HPLC) analysis of $H V C 1$. HVC1 $(100 \mathrm{mg})$ was precisely weighed and dissolved in $10 \mathrm{ml}$ methanol (HPLC grade, J.T. Baker Co. Ltd., Center Valley, PA, USA), followed by filtration through a $0.45-\mu \mathrm{m}$ polyvinylidene difluoride syringe filter (Waters, Corporation, Milford, CT, USA). Standards used in the HPLC analysis of $\mathrm{HVCl}$ were as follows: Sennoside A and sennoside B (Rhubarb standards; Sigma-Aldrich); coptisine, berberine and wogonin (C.chinensis standards; Sigma-Aldrich); baicalin, and baicalein (S. baicalensis standards; Sigma-Aldrich); prunetin ( $P$. yedoensis standard; Sigma-Aldrich); and genistein-7-glucose and prunetin-5-glucose $(P$. yedoensis standards; directly isolated). The standards (1 $\mathrm{mg}$ ) were dissolved at a concentration of $100 \mu \mathrm{g} / \mathrm{ml}$, and the HPLC chromatograms of the standards were obtained. The HPLC apparatus was a Gilson system equipped with a 234 Autosampler, a UV/VIS-155 detector, and a 321 HPLC Pump (Gilson, Seoul, Korea). A Luna $4.60 \times 264 \mathrm{~mm} \mathrm{C}_{18}$ reversed-phase column with 5-mm particles (Phenomenex, CA, USA) was used. The mobile phase consisted of $0.1 \%$ formic acid (A) and acetonitrile (HPLC grade, J.T. Baker Co. Ltd.) (B) in a ratio specified by the following binary gradient with linear interpolation: 0 min $20 \%$ B, 60 min $30 \%$ B, 70 min $60 \%$ B and 100 min $70 \%$ B. The column eluent was monitored at UV $250 \mathrm{~nm}$, following which all solvents were degassed with a micromembrane filter (Advantec, Tokyo, Japan). Chromatography was performed at room temperature at a flow rate of $0.5 \mathrm{ml} / \mathrm{min}$, and $10 \mu \mathrm{l}$ was analyzed for $100 \mathrm{~min}$.

Statistical analysis. All the values are expressed as the mean \pm standard error of the mean. Data were analyzed using one-way analysis of variance with Dunnett's test. Statistical analysis was performed using GraphPad Prism (version 5; Graphpad Software Inc., La Jolla, CA, USA). P $<0.05$ was considered to indicate a statistically significant difference.
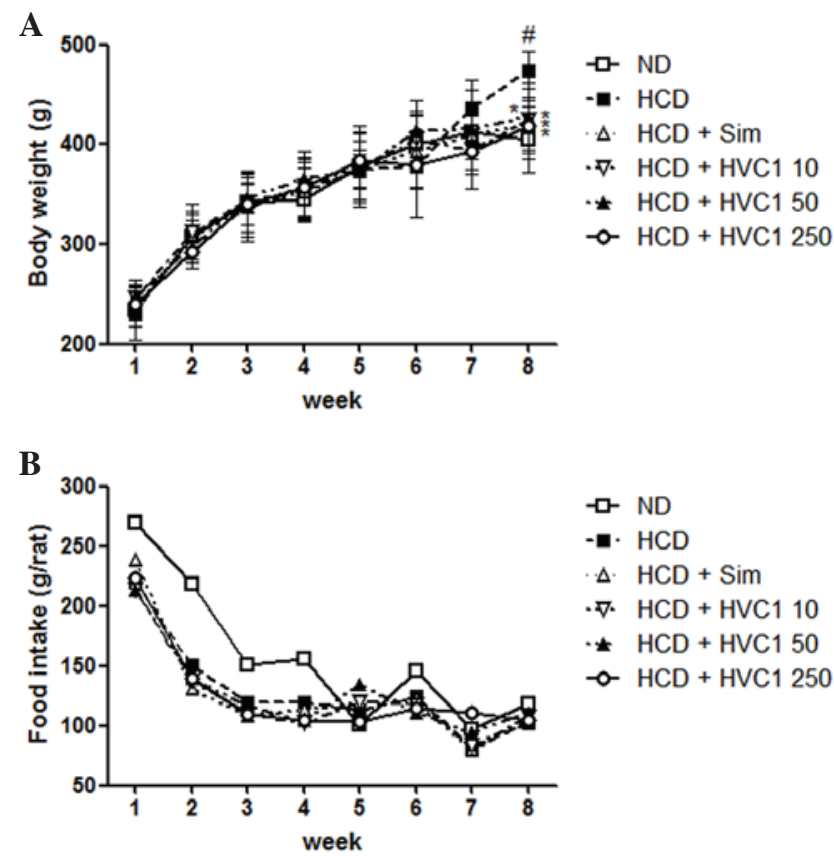

Figure 1. Effects of $\mathrm{HVCl}$ on body weight and food intake in rats. (A) Body weight and (B) food intake were recorded weekly. The values are represented as the mean \pm standard error of the mean $(\mathrm{n}=6) .{ }^{\#} \mathrm{P}<0.05$, compared with the ND group; ${ }^{*} \mathrm{P}<0.05$ compared with the HCD group. ND, normal diet group; HCD, high-cholesterol diet group; Sim, simvastatin $(10 \mathrm{mg} / \mathrm{kg})$ treated with $\mathrm{HCD}$ group; $\mathrm{HVCl}$, $\mathrm{HVCl}(50,100$, or $250 \mathrm{mg} / \mathrm{kg})$ treated with $\mathrm{HCD}$ group.

\section{Results}

Effects of HVCl on body weight gains. There was a significant difference in body weight between the ND and HCD groups. The HVC1-treated groups had a significantly lower weight when compared with the HCD group, as shown in Fig. 1. The HCD group gained an average of $202.5 \mathrm{~g}$, whereas the HVC1 treated groups $(10,50$, or $250 \mathrm{mg} / \mathrm{kg})$ gained only 160.2 , 176.6 or $172.2 \mathrm{~g}$, respectively, after 8 weeks (Fig. 1A). During the experimental period, there were no significant differences in food and water intake in the HCD group compared with the other treatment groups (Fig. 1B).

Effects of HVCl on serum cholesterol levels. To determine the effect of HVC1 on HCD-induced changes in serum cholesterol levels, serum samples were prepared from each group and cholesterol levels were analyzed by enzymatic methods. 
The concentrations of serum TC and LDL cholesterol were significantly increased by a HCD. Administration of HVC1 significantly and dose-dependently suppressed the elevation of serum TC and LDL cholesterol levels (Fig. 2A and B). The serum level of TC in the HCD group was $443.3 \pm 67.7 \mathrm{mg} / \mathrm{dl}$, and 10,50 and $250 \mathrm{mg} / \mathrm{kg} \mathrm{HVC1}$ reduced TC levels to $255.0 \pm 41.6$, $218.0 \pm 34.6$ and $170.3 \pm 21.0 \mathrm{mg} / \mathrm{dl}$, respectively (Fig. 2A). The serum level of LDL cholesterol in the HCD group was $124.3 \pm 23.7 \mathrm{mg} / \mathrm{dl}$ and 10,50 and $250 \mathrm{mg} / \mathrm{kg} \mathrm{HVC1}$ reduced LDL levels to $80.7 \pm 11.6,67.0 \pm 6.9$ and $49.7 \pm 11.0 \mathrm{mg} / \mathrm{dl}$, respectively (Fig. 2B). There was a dose-dependent response with HVC1 for TC and LDL cholesterol levels; HVC1 exhibited a greater effect than that of simvastatin. In addition, the administration of $\mathrm{HVC} 1(250 \mathrm{mg} / \mathrm{kg})$ also significantly reversed the HCD-induced reduction of serum HDL cholesterol levels (Fig. 1C). Serum levels of HDL cholesterol in the HCD group and $\mathrm{HVC} 1$ group $(250 \mathrm{mg} / \mathrm{kg})$ were $14.7 \pm 3.1$ and $24.7 \pm 2.9 \mathrm{mg} / \mathrm{dl}$, respectively (Fig. 2C). Based on this data, HVC1 more effectively inhibited HCD-induced changes in serum cholesterol levels than the hypolipidemic drug, simvastatin.

Effects of HVCl on lipid accumulation in the liver and aorta. To identify the effect of HVC1 on HCD-induced lipid accumulation in the liver and aorta, tissue samples were prepared from each group and stained with H\&E or Oil red O. As shown in Fig. 3 the H\&E staining results for liver tissues demonstrated that in the HCD group, lipid droplets appeared as small vacuoles within liver cells. Enlargement of lipid droplets was more pronounced in the liver tissue of rats in the HCD group than the HVC1 group $(250 \mathrm{mg} / \mathrm{kg})$. In addition, lipid accumulation in the aorta was more visible in the HCD group than in the HVC1-treated groups (Fig. 4). Thus, the results for each representative tissue clearly showed that lipid accumulation in the liver and aorta was higher in the HCD group than in the HVC1 group $(250 \mathrm{mg} / \mathrm{kg})$.

Effects of HVCl on the mRNA expression of hepatic PPAR- $\gamma$, $H M G-C O A R$ and LDLR. To investigate the effects of HVC1 on the mRNA expression of hepatic PPAR- $\gamma$, HMG-CoA R and LDLR, RT-qPCR was performed. As shown in Fig. 5A and $B$, the mRNA expression of PPAR $-\gamma$ and HMG-CoA R was upregulated in the HCD group as compared with the ND group, and administration of HVC1 significantly and dose-dependently suppressed the mRNA expression of PPAR- $\gamma$ and HMG-CoA R. In addition, administration of HVC1 $(250 \mathrm{mg} / \mathrm{kg})$ significantly reversed the HCD-induced reduction of LDLR mRNA expression (Fig. 5C). Notably, $\mathrm{HVC1}$ had a more potent effect than simvastatin.

Effects of HVCl on the activation of AMPK. Since AMPK has been implicated in lipid metabolism (17), it was investigated whether HVC1 could inhibit HCD-induced dephosphorylation of AMPK. Phosphorylation and total protein levels of AMPK were decreased by HCD and HVC1 significantly reversed these effects (Fig. 5D).

Standard material analysis. For standardization, HPLC analysis was performed. The retention time of the sample mixture was as follows: 3.49 min for sennoside A and sennoside B, 4.98 min for genistein-7-glucose, $9.61 \mathrm{~min}$ for coptisine, $13.78 \mathrm{~min}$ for baicalin, $17.18 \mathrm{~min}$ for prunetin-5-glucose,
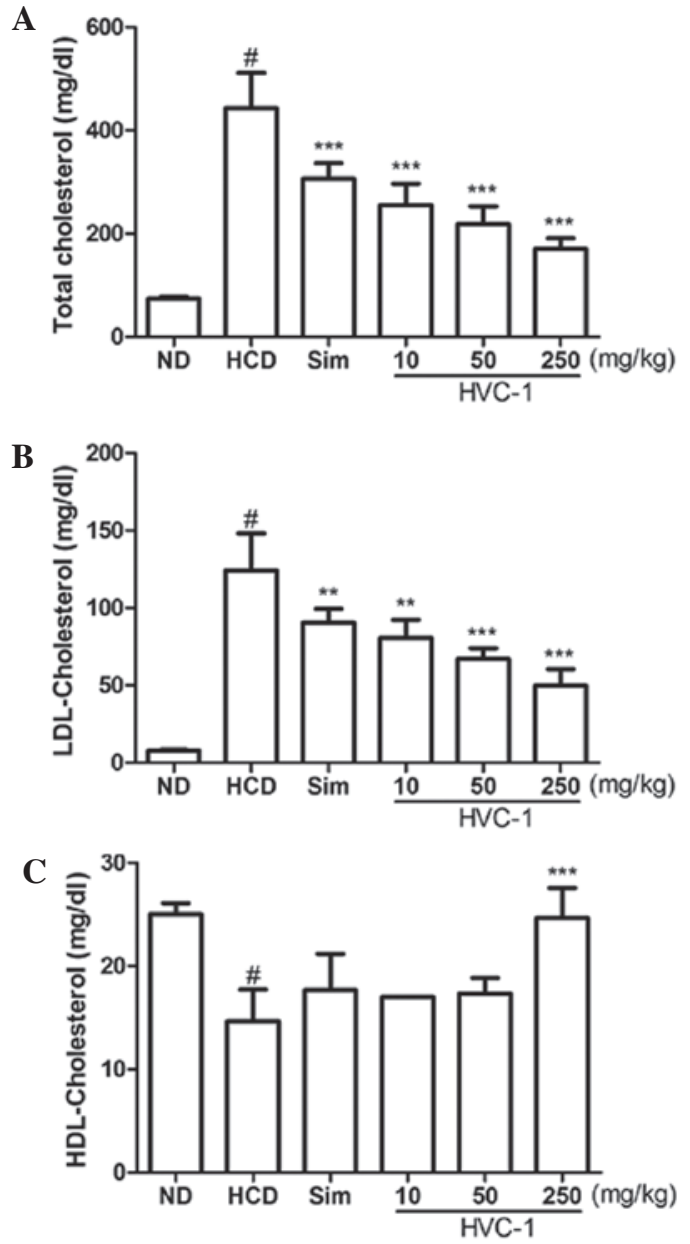

Figure 2. Effects of HVC1 on serum cholesterol in high-cholesterol diet fed rats. Serum concentrations of TC, LDL-cholesterol and HDL-cholesterol were determined by enzymatic methods. (A) Serum level of TC. (B) Serum level of LDL-cholesterol. (C) Serum level of HDL-cholesterol. ND, normal diet group; HCD, high-cholesterol diet group; Sim, simvastatin $(10 \mathrm{mg} / \mathrm{kg})$ treated with HCD group; HVC1, HVC1 treated with HCD group. The values are presented as the mean \pm standard error of the mean $(n=6)$. ${ }^{\#} \mathrm{P}<0.05$, compared with $\mathrm{ND} ;{ }^{* *} \mathrm{P}<0.01$ compared with $\mathrm{HCD} ;{ }^{* * * *} \mathrm{P}<0.001$ compared with HCD.

$21.22 \mathrm{~min}$ for berberine, $59.76 \mathrm{~min}$ for baicalein, $72.53 \mathrm{~min}$ for wogonin, $74.12 \mathrm{~min}$ for prunetin (Fig. 6A and B).

\section{Discussion}

In the present study, the hypolipidemic effects of $\mathrm{HVC1}$ against HCD-induced hyperlipidemia in rats were investigated. HVC1 significantly reduced serum lipid levels and inhibited the expression of PPAR- $\gamma$, HMG-CoA R and LDLR.

Increased serum concentrations of LDL cholesterol and triglycerides are atherogenic and have been recognized as a risk factor for cardiovascular diseases (18). An increased HDL level has been considered cardioprotective. In this study, HVC1 decreased TC and LDL, and increased HDL in the serum of $\mathrm{HCD}$ fed rats. In the same manner, lipid accumulation in the aorta was less visible in the HVC1-treated groups than in the HCD group. HVC1 consists of $R$. palmatum rhizome, $P$. yedoensi bark, $C$. chinensis rhizome and $S$. baicalensis radix, all of which are known to positively affect lipid metabolism. According to 


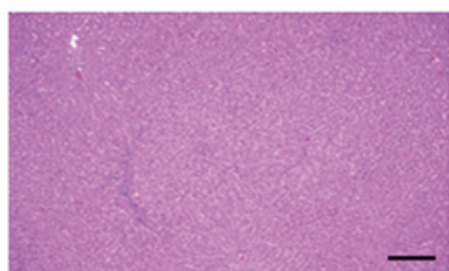

$\mathrm{ND}$

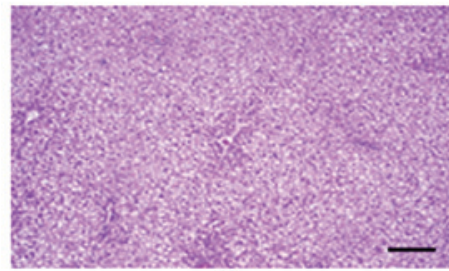

10

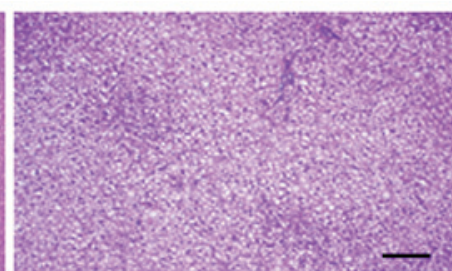

HCD

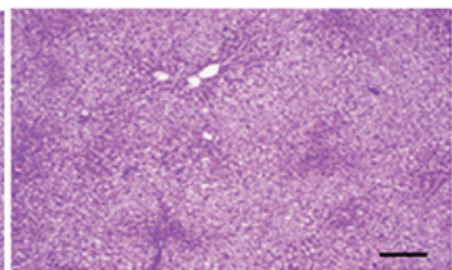

50

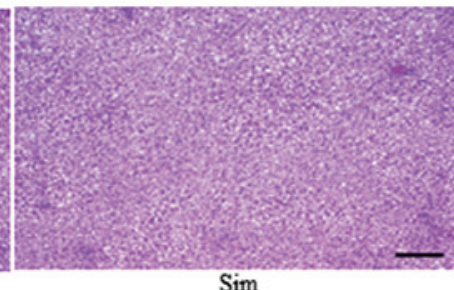

$\operatorname{Sim}$

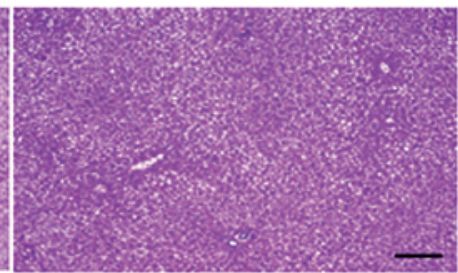

250

HVC-1 $(\mathrm{mg} / \mathrm{kg})$

Figure 3. Effects of HVC1 on lipid accumulation in the liver. Liver from a representative rat in each group was fixed, embedded in paraffin and stained with hematoxylin and eosin. Images are taken at the original magnification of x100. Scale bar, $100 \mathrm{~mm}$. ND, Normal diet group; HCD, High-cholesterol diet group; Sim, Simvastatin $(10 \mathrm{mg} / \mathrm{kg}$ ) treated with HCD group; HVC1, HVC1 treated with HCD group.

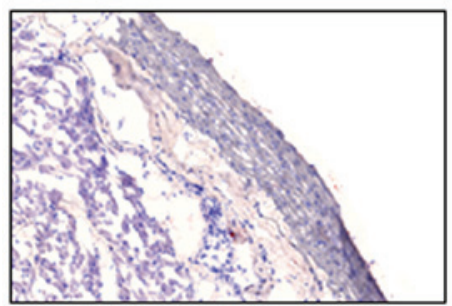

$\mathrm{ND}$

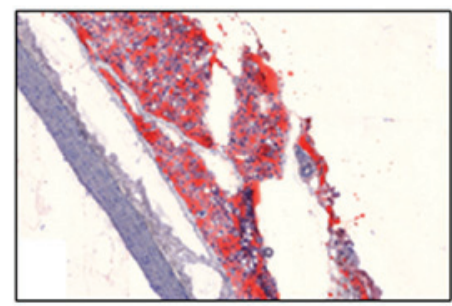

10

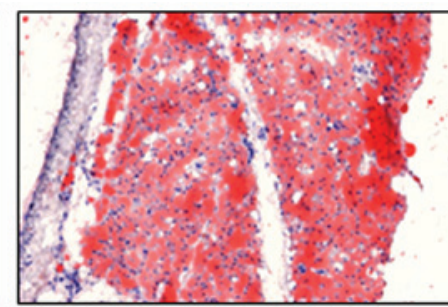

HCD

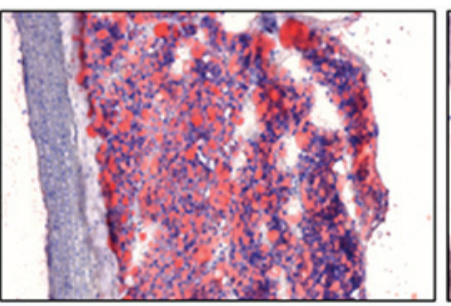

50

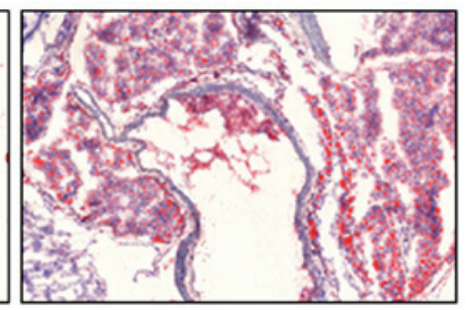

$\operatorname{Sim}$

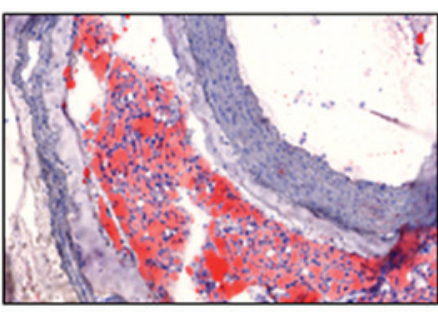

250

HVC-1 (mg/kg)

Figure 4. Effects of $\mathrm{HVCl}$ on lipid accumulation in the aorta. Aorta from a representative rat in each group was fixed, embedded in paraffin and stained with Oil red O. Images are taken at the original magnification of x100. ND, Normal diet group; HCD, High-cholesterol diet group; HVC1, HVC1 treated with HCD group.

the present study, these four herbs or active compounds may be associated with the powerful effect of HVC1.

Generally, the liver is considered an essential organ in lipid metabolism. As shown in Fig. 3, HCD induced lipid accumulation in the liver tissue, but treatment with HVC1 suppressed lipid droplet content in liver tissue. Hepatic lipid metabolism is a highly co-ordinated process, in which numerous pathways are regulated by transcription (19). PPAR- $\gamma$ is a nuclear receptor and ligand-activated transcription factor that is involved in the expression of lipogenic enzymes, such as acetyl CoA carboxylase and fatty acid synthetase (20). HMG-CoA R is a transmembrane protein that is implicated in the synthesis of lipids. It is the rate-limiting step in cholesterol synthesis and represents the major target for the cholesterol-lowering drugs, statins (21).
Inhibition of the HMG-CoA R induces the expression of LDLR in the liver (22). LDLR is a cell-surface receptor that increases the catabolism of plasma LDL and lowers the plasma concentration of cholesterol. LDL-cholesterol binds to the LDLR, is internalized in a process known as endocytosis, and prevents the LDL diffusing around the membrane surface (23). Endocytosis occurs predominantly in the liver, which removes $\sim 70 \%$ of LDL from the circulation (24). In this study, the mRNA expression of PPAR- $\gamma$, HMG-CoA R and LDLR were significantly and dose-dependently recovered by HVC1. These results indicate that $\mathrm{HVC1}$ could reduce serum lipid levels and fat accumulation in the liver and aorta through the regulation of gene expression.

AMPK is a well-known regulator of lipid metabolism in the liver, and is also involved in cellular energy homeostasis (19). 

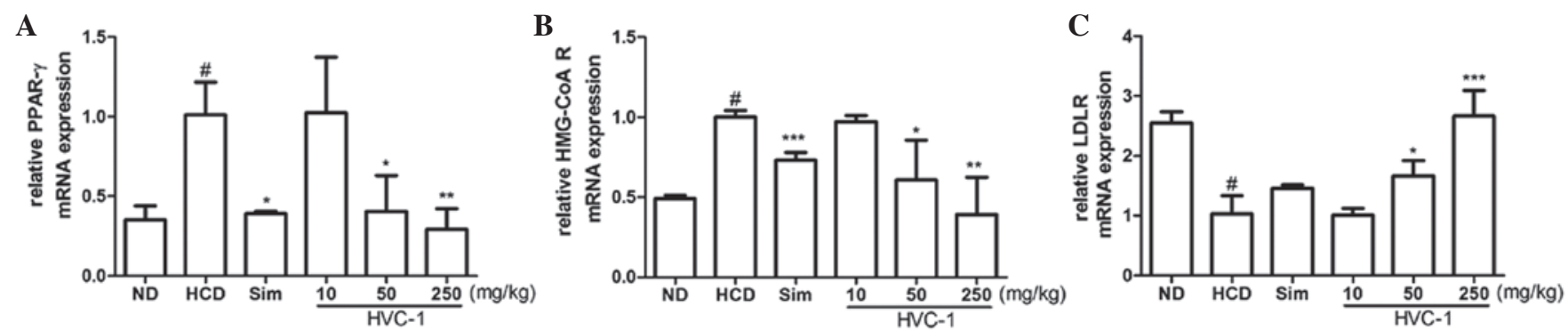

D
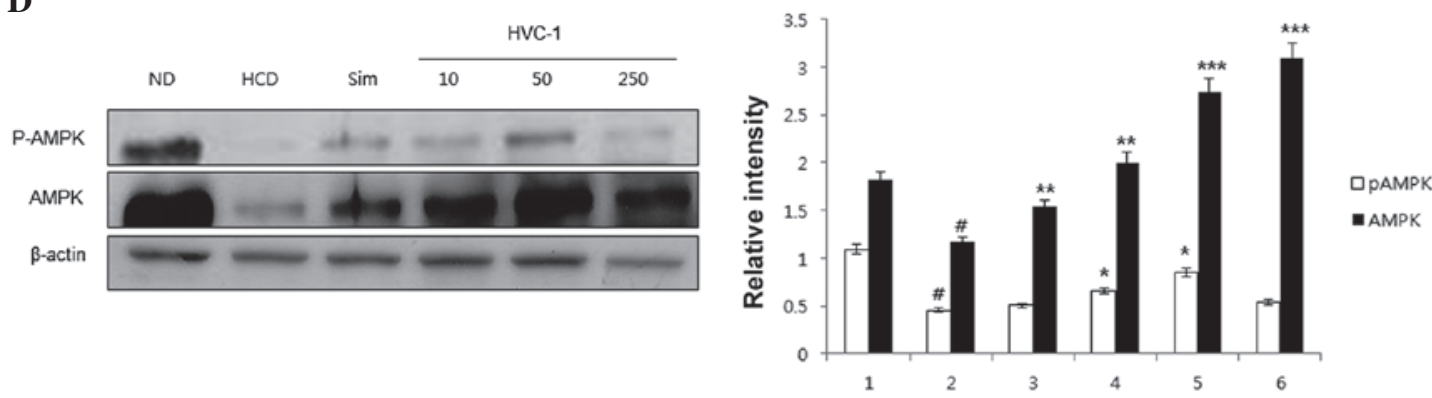

Figure 5. Effects of HVC1 on mRNA expression of PPAR- $\gamma$, HMG-CoA R and LDLR, and the activation of AMPK in the liver tissue. Total RNA was prepared for the real-time-PCR analysis of (A) PPAR- $\gamma$, (B) HMG-CoA R and (C) LDLR gene expression from liver tissues. Reverse transcription-quantitative polymerase chain reaction analysis was conducted using a Step One Plus Real-time PCR system. (D) The liver tissue was homogenized and total protein was prepared. Western blot analysis was performed using specific antibodies. $\beta$-actin was used as an internal control. ND, Normal diet group; HCD, High-cholesterol diet group; Sim, Simvastatin $(10 \mathrm{mg} / \mathrm{kg})$ treated with $\mathrm{HCD}$ group; $\mathrm{HVC1}, \mathrm{HVCl}$ treated with $\mathrm{HCD}$ group. The values are represented as the mean \pm standard error of the mean $(n=6) .{ }^{*} \mathrm{P}<0.05$, compared to $\mathrm{ND} ;{ }^{*} \mathrm{P}<0.05,{ }^{* * *} \mathrm{P}<0.01$ and ${ }^{* * * *} \mathrm{P}<0.001$ compared to HCD. PPAR- $\gamma$, peroxisome proliferator-activated receptor- $\gamma$; HMG-CoA R, 3-hydroxy-3-methylglutaryl-CoA reductase; LDLR, low-density lipoprotein receptor; AMPK, AMP-activated protein kinase; p-, phosphorylated.

A

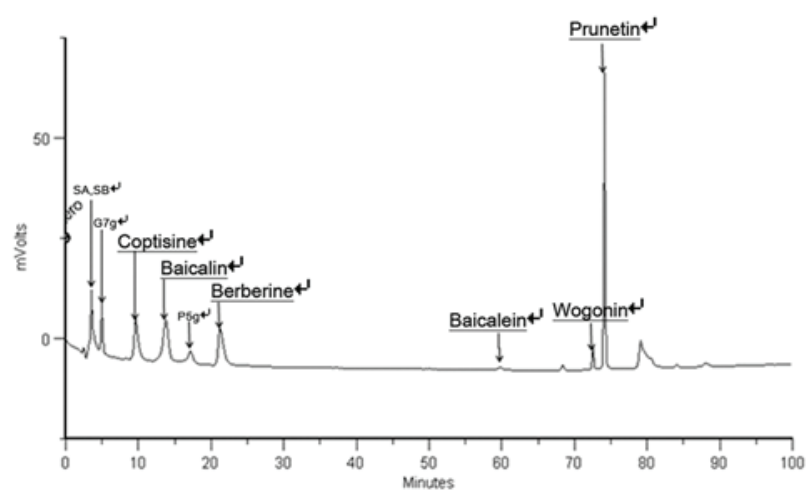

B

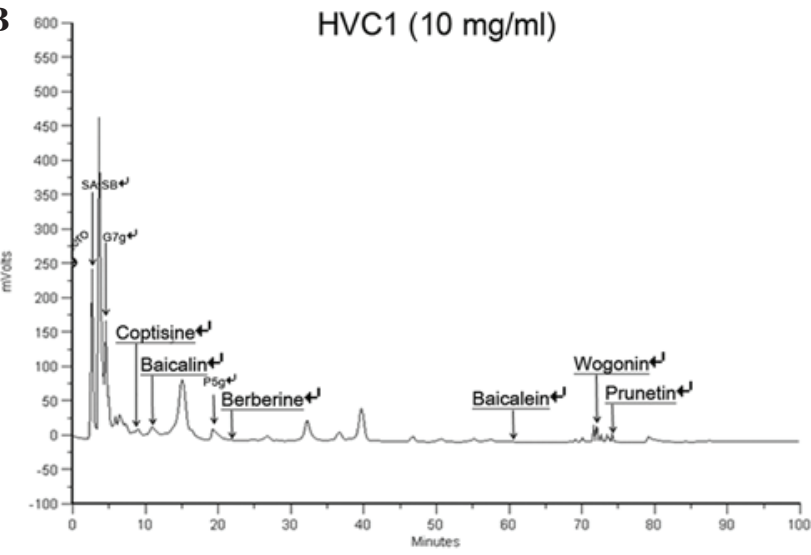

Figure 6. HPLC chromatogram of HVC1. (A) HPLC chromatogram of HVC1 standard mixtures. (B) HPLC chromatogram of HVC1. SA, sennoside A; SB, sennoside B; G7g, genistein-7-glucose; P5g, prunetin-5-glucose.

It is known that AMPK is regulated by phosphorylation and inactivates HMG-CoA R, a key enzyme in cholesterol synthesis in the liver (25). To investigate a possible mechanism for the hypolipidemic effects of HVC1, the phosphorylation and total protein level of AMPK were examined. HVC1 significantly reversed the reduction of AMPK phosphorylation, as well as the reduction of total AMPK protein levels. This data indicates that $\mathrm{HVC} 1$ could regulate AMPK at a transcriptional or translational level. Therefore, it may also be possible that $\mathrm{HVC1}$ exerts its hypolipidemic effects through the regulation of AMPK.

In conclusion, $\mathrm{HVC} 1$ effectively suppressed serum lipid levels and fat accumulation in the liver and aorta of rats with
HCD-induced hyperlipidemia. The mechanisms underlying the hypolipidemic effect of $\mathrm{HVCl}$ appear to involve the recovery of PPAR- $\gamma$, HMG-CoA R and LDLR expression through the induction of AMPK. The findings clearly demonstrate that HVC1 has a potent hypolipidemic effect, and suggests that $\mathrm{HVC1}$ should be evaluated as a potential treatment for hyperlipidemia.

\section{Acknowledgements}

This study was supported by a grant from the Korea Healthcare Technology R\&D Project, Ministry of Health \& Welfare, Republic of Korea (grant no. B110081). 


\section{References}

1. Saha SA and Arora RR: Hyperlipidaemia and cardiovascular disease: Do fibrates have a role? Curr Opin Lipidol 22: 270-276, 2011.

2. Ahn D: Illustrated book of Korean medicinal herbs. Kyohak Publishing, 1998.

3. Kim J: Illustrated natural drugs encyclopedia, colorth edition ed. Namsandang, 1997.

4. Lee K, Ham I, Yang G, Lee M, Bu Y, Kim H and Choi HY: Vasorelaxant effect of Prunus yedoensis bark. BMC Complement Altern Med 13: 31, 2013.

5. Ahn TG, Yang G, Lee HM, Kim MD, Choi HY, Park KS, Lee SD, Kook YB and An HJ: Molecular mechanisms underlying the anti-obesity potential of prunetin, an $\mathrm{O}$-methylated isoflavone. Biochem Pharmacol 85: 1525-1533, 2013.

6. Liu Q, Zhang XL, Tao RY, Niu YJ, Chen XG, Tian JY and Ye F: Rhein, an inhibitor of adipocyte differentiation and adipogenesis. J Asian Nat Prod Res 13: 714-723, 2011.

7. Huang Q, Lu G, Shen HM, Chung MC and Ong CN: Anti-cancer properties of anthraquinones from rhubarb. Med Res Rev 27: 609-630, 2007.

8. Guo MZ, Li XS, Xu HR, Mei ZC, Shen W and Ye XF: Rhein inhibits liver fibrosis induced by carbon tetrachloride in rats. Acta Pharmacol Sin 23: 739-744, 2002.

9. Choi SB, Ko BS, Park SK, Jang JS and Park S: Insulin sensitizing and alpha-glucoamylase inhibitory action of sennosides, rheins and rhaponticin in Rhei Rhizoma. Life Sci 78: 934-942, 2006.

10. Sheng X, Wang M, Lu M, Xi B, Sheng H and Zang YQ: Rhein ameliorates fatty liver disease through negative energy balance, hepatic lipogenic regulation, and immunomodulation in diet-induced obese mice. Am J Physiol Endocrinol Metab 300 E886-E893, 2011.

11. Xie W, Gu D, Li J, Cui K and Zhang Y: Effects and action mechanisms of berberine and Rhizoma coptidis on gut microbes and obesity in high-fat diet-fed C57BL/6J mice. PLoS One 6 : e24520, 2011.

12. Xie HC and Shang J: Study on the extraction process of total anthraquinones in Radix et Rhizoma Rhei and their antilipemic effects. Afr J Tradit Complement Altern Med 11: 358-362, 2014.
13. Lee IS, Park S, Park K and Choue R: Hepatoprotective activity of scutellariae radix extract in mice fed a high fat diet with chronic alcohol exposure. Phytother Res 25: 1348-1353, 2011.

14. Bak EJ, Kim J, Choi YH, Kim JH, Lee DE, Woo GH, Cha JH and Yoo YJ: Wogonin ameliorates hyperglycemia and dyslipidemia via PPAR $\alpha$ activation in $\mathrm{db} / \mathrm{db}$ mice. Clin Nutr 33: 156-163, 2014.

15. Nunnari JJ, Zand T, Joris I and Majno G: Quantitation of oil red $\mathrm{O}$ staining of the aorta in hypercholesterolemic rats. Exp Mol Pathol 51: 1-8, 1989.

16. Lee K, Kim B, Hur H, Chinannai KS, Ham I and Choi HY: Antihypertensive effect of the GaMiSamHwangSaSimTang in spontaneous hypertensive rats. Evid Based Complement Alternat Med 2015: 802368, 2015

17. Unger RH: The hyperleptinemia of obesity-regulator of caloric surpluses. Cell 117: 145-146, 2004

18. Boruah DC, Devi R, Tamuli S, Kotoky J and Sharma DK: Hypolipidemic activity of crude polyphenols from the leaves of Clerodendron colebrookianum Walp in cholesterol fed rats. J Food Sci Technol 51: 3333-3340, 2014.

19. Jump DB, Tripathy S and Depner CM: Fatty acid-regulated transcription factors in the liver. Annu Rev Nutr 33: 249-269, 2013.

20. Walczak R and Tontonoz P: PPARadigms and PPARadoxes: Expanding roles for PPARgamma in the control of lipid metabolism. J Lipid Res 43: 177-186, 2002.

21. Istvan ES and Deisenhofer J: Structural mechanism for statin inhibition of HMG-CoA reductase. Science 292: 1160-1164, 2001.

22. Rudling M: Hepatic mRNA levels for the LDL receptor and HMG-CoA reductase show coordinate regulation in vivo. J Lipid Res 33: 493-501, 1992.

23. Südhof TC, Goldstein JL, Brown MS and Russell DW: The LDL receptor gene: A mosaic of exons shared with different proteins. Science 228: 815-822, 1985.

24. Pieper-Fürst U and Lammert F: Low-density lipoprotein receptors in liver: Old acquaintances and a newcomer. Biochim Biophys Acta 1831: 1191-1198, 2013.

25. Lim CT, Kola B and Korbonits M: AMPK as a mediator of hormonal signalling. J Mol Endocrinol 44: 87-97, 2010. 